\section{Kidney Blood Pressure Research}

\title{
Depression and Physical Frailty Have Additive Effect on the Nutritional Status and Clinical Outcome of Chinese Peritoneal Dialysis
}

\author{
Cheuk-Chun Szeto Gordon Chun-Kau Chan Jack Kit-Chung Ng \\ Kai-Ming Chow Bonnie Ching-Ha Kwan Phyllis Mei-Shan Cheng \\ Vickie Wai-Ki Kwong Man-Ching Law Chi-Bon Leung Philip Kam-Tao Li \\ Carol \& Richard Yu Peritoneal Dialysis Research Centre, Department of Medicine \& Therapeutics, The \\ Chinese University of Hong Kong, Shatin, Hong Kong, China
}

\section{Key Words}

Renal failure • Survival • Psychiatry

\begin{abstract}
Background/Aims: Frailty and depression both contribute to malnutrition and adverse clinical outcome of peritoneal dialysis (PD) patients. However, their interaction is incompletely defined. Methods: We studied 178 adult Chinese PD patients. Physical frailty was assessed by a validated in-house questionnaire; depressive symptoms was screened by the Geriatric Depression Scale; nutritional status was determined by subjective global assessment (SGA) and malnutrition inflammation score (MIS). All patients were followed for up to 24 months for survival and hospitalization analysis. Results: There were 111 patients (62.4\%) physically frail, amongst those $48(43.2 \%)$ had depressive symptoms. Only 1 patient had depressive symptoms without frailty. There was an additive effect of depressive symptoms and physical frailty on nutritional status. For the groups with no frailty, frail but no depressive symptoms, and frail with depressive symptoms, serum albumin decreased in a stepwise manner (35.8 $\pm 5.6,34.9 \pm 4.4$, and $32.9 \pm 5.3 \mathrm{~g} / \mathrm{L}$, respectively, $\mathrm{p}=0.025)$; overall SGA score was $5.75 \pm$ $0.61,5.41 \pm 0.59$, and $5.04 \pm 0.77$, respectively $(p<0.0001$ ), and MIS was $5.12 \pm 2.30,7.13$ \pm 3.22 , and $9.48 \pm 3.97$, respectively $(p<0.0001)$. At 24 months, patient survival was $86.6 \%$, $71.4 \%$, and $62.5 \%$ for patients with no frailty, frail but no depressive symptoms, and frail with depressive symptoms, respective $(p=0.001)$. The median number of hospital stay was 8.04 (inter-quartile range [IQR] 0.91 - 19.42), 14.05 (IQR 3.57 - 37.27), and 26.62 (IQR 10.65 - 61.18) days per year of follow up, respectively $(p<0.0001)$. Conclusion: Physical frailty and depressive
\end{abstract}




\section{Kidney Blood Pressure Research}

symptoms are both common in Chinese PD patients, and they have additive adverse effect on the nutritional status and clinical outcome.

(C) 2018 The Author(s)

Published by S. Karger AG, Basel

\section{Introduction}

The number of peritoneal dialysis (PD) patient is growing quickly around the world [1]. Although the overall survival of PD patients improved substantially in the past decades [2], PD patients nowadays often have multiple comorbid diseases and represent a heavy burden to the health care system [3].

In addition to organ-system specific comorbid diseases, it is increasingly recognized that physical frailty, depression, and cognitive impairment are important contributing factors to the adverse clinical outcome of chronic kidney disease (CKD) and dialysis patients [4, 5]. Frailty is defined as a clinically recognizable state of increased vulnerability resulting from aging-associated decline in reserve and function across multiple physiologic systems, such that the ability to cope with every day or acute stressors is comprised [6]. Traditionally, frailty refers to a clinical syndrome involving unintentional weight loss, self-reported exhaustion, weakness, slow walking speed, and low physical activity [7]. It is present in around $35 \%$ of hemodialysis patients [8] and, regardless of age, frailty is a strong independent predictor of mortality and number of hospitalizations $[8,9]$. Cognitive impairment is present in over $20 \%$ of dialysis patients $[9,10]$, and is associated with increased risks of mortality and adverse clinical outcome $[9,11]$. Similarly, depressive symptoms are present in over $50 \%$ of PD patients [10] and is associated with excessive morality and represents a risk factor of peritonitis $[12,13]$.

Physical frailty, depression, and cognitive impairment are inter-linked. Cognitive impairment is often taken as an intrinsic domain of frailty [14]. In adult incident hemodialysis patients, frailty is associated with cognitive problems [15]. A number of underlying factors, such as vascular changes, inflammation, insulin resistance, and nutrition, contribute to the development of both physical frailty and cognitive impairment, and represent the underlying mechanisms behind this link [14]. Furthermore, physical frailty is also associated with new onset cognitive impairment in community-based elderly persons [16], and a causative role has been proposed [17]. On the other hand, depression is sometimes difficult to be discerned from frailty in the elderly. In community-dwelling people, depression and frailty are independent and probably additive risk factors for mortality and other adverse health outcomes [18-20]. A recent systematic review reported a consistent relationship between depression and new onset physical frailty [21]. Similarly, depression has been reported to be closely associated with cognitive impairment in PD patients [10]. However, the intricate three-way internal relationship between frailty, depression, and cognitive impairment remains incompletely defined. Herein we present a prospective study that aims to dissect the internal relationship between frailty and depression, and their contribution to malnutrition and adverse clinical outcome in Chinese PD patients.

\section{Materials and Methods}

\section{Patient selection}

This study was approved by the Joint Chinese University Hong Kong-New Territories East Cluster Clinical Research Ethics Committee. All procedures were in adherence to the Declaration of Helsinki. We recruited 178 adult Chinese PD patients from the dialysis clinic of our hospital from April to May 2013. We excluded patients who were unlikely to survive for 6 months, who were planned to have elective living donor kidney transplant or transfer to other renal centers within 6 months. Nutritional status and dialysis adequacy were assessed at the same time. Baseline clinical and laboratory data were obtained by chart 


\section{Kidney Blood Pressure Research}

Szeto et al.: Depression and Frailty in PD

review. The severity of comorbidity load was quantified by the modified Charlson's Comorbidity Index as previously described [22].

\section{Assessment of frailty, depression, and cognitive impairment}

After informed consent, physical frailty, depressive symptoms and cognitive function were assessed by standard instruments as described previously [23-25]. Briefly, frailty was assessed by an in-house Chinese questionnaire that was validated by our group. The frailty questionnaire consists of 30 dichotomous questions that covers various aspects of physical functioning and self-care capability [23]. In this questionnaire, physical frailty was defined as a score of 6 or above [23].

Depressive symptoms were screened by a validated Chinese version of the Geriatric Depression Scale (GDS) [24], which is a standardized self-reported questionnaire that consists of 15 dichotomous items. For GDS, substantial depressive symptom was defined as a score 8 or above. Cognitive impairment was assessed by a validated Chinese version of the Abbreviated Mental Test (AMT), which is a standardized questionnaire that consists of 10 dichotomized items [25]. For AMT, cognitive impairment was defined as a score of 7 or below.

\section{Assessment of nutrition and dialysis adequacy}

Dialysis adequacy and residual glomerular filtration rate (GFR) was assessed by 24-hour urine and dialysate collection [26]. Nutritional status was represented by serum albumin level, subjective global assessment (SGA), comprehensive malnutrition-inflammation score (MIS), and normalized protein nitrogen appearance (NPNA). For SGA, the 4-item 7-point scoring system validated in PD patients was used [27]. The calculation of MIS was described previously [28]. Briefly, MIS consists of 4 main parts and 10 components, all scored from 0 (normal) to 3 (very severe). The total score ranged from 0 to 30. NPNA was calculated by the modified Bergstrom's formula [29].

\section{Body composition}

The method of determining body composition by bioimpedance spectroscopy has been described previously [30]. Briefly, electrodes were attached to one hand and one foot with the patient in a supine position. After patient cable was connected, the measurement would complete automatically in 2 minutes. In the present study, we computed the following parameters: extracellular water (ECW), lean tissue mass, adipose tissue mass, and volume of over hydration.

\section{Clinical outcome}

All patients were followed for up to 24 months. The clinical management was decided by individual clinician and not affected by the study. We determined patient survival and technique survival. Technique failure was defined as transfer to chronic hemodialysis because Hong Kong practices the "PD-first" policy, and patients would only be transferred to chronic hemodialysis if PD fails [31]. Kidney transplant was censored for technique survival analysis. Censoring events for technique survival include recovery of renal function, loss to follow up, or transfer to other dialysis centers. We also determined the number of hospital admission and duration of hospitalization during the study period.

\section{Statistical analysis}

Statistical analysis was performed by SPSS for Windows software version 18.0 (SPSS Inc., Chicago, IL). Data were expressed as means $\pm \mathrm{SD}$, except that data on hospitalization were expressed as median (inter-quartile range [IQR]) as they were highly skewed. Data were compared by Student's t test, Chi square test or Kruskal Wallis test as appropriate. Since cognitive impairment was found to be uncommon in our cohort, and most patients with depressive symptoms were also physically frail, we divided the patients into 3 groups for further analysis: no frailty, frail but no depressive symptoms, and frail with depressive symptoms. As the data on hospital admission and duration of hospitalization are highly skewed, they were compared between groups by the Mann Whitney U test. Survival data are presented in the Kaplan Meier plot and compared between groups by $\log$ rank test. A value of $\mathrm{p}<0.05$ was considered statistically significant. All probabilities were two-tailed. 


\section{Kidney Blood Pressure Research}

\section{Results}

We studied 178 patients; 111 (62.4\%) were physically frail, 49 (27.5\%) had depressive symptoms, and 14 (7.9\%) had cognitive impairment. There was substantial overlap amongst these 3 groups of patients (Fig. 1). Their baseline clinical and demographic data are summarized and compared in Table 1.

\section{Nutritional status}

The baseline biochemical and nutritional data are summarized and compared in Table 2. In essence, frail patients were older, were dialyzed longer, had higher Charlson's comorbidity score, lower residual GFR, and worse nutritional status (i.e. lower SGA score and higher MIS) than other patients (see Tables 1 and 2). Bioimpedance spectroscopy showed that although patients with and without frailty had similar body weight, frail patients had lower lean tissue mass but higher adipose tissue mass than the others (see Table 2).

Similarly, patients with cognitive impairment were older, had higher Charlson's comorbidity score, and higher MIS than those without (see Tables 1 and 2). Bioimpedance spectroscopy also showed that patients with cognitive impairment had lower lean tissue mass but higher adipose tissue mass than the others (see Table 2).

On the other hand, patients with depressive symptoms had lower serum albumin and worse nutritional status (i.e. lower SGA score and higher MIS) than those without (Table 2). Patients with depressive symptoms also had more overhydration as revealed by bioimpedance spectroscopy, although their lean tissue mass and adipose tissue mass were similar to those without depression (Table 2).

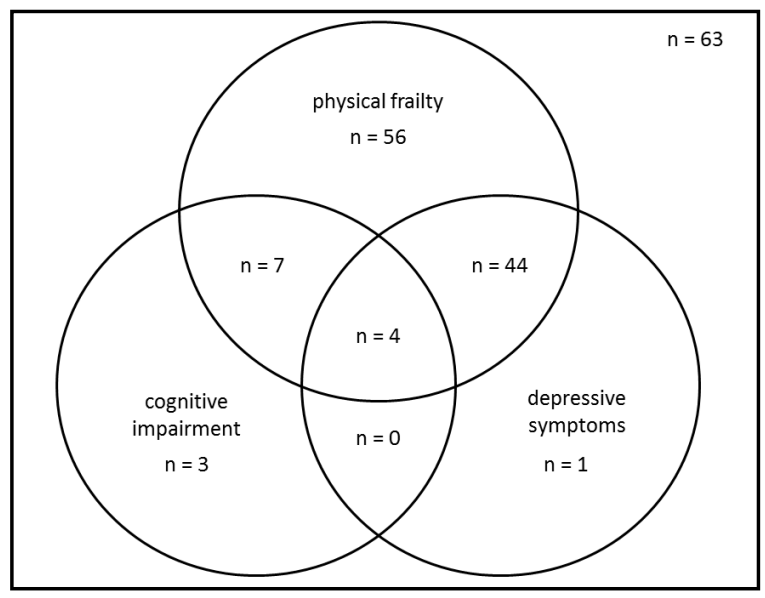

Fig. 1. Venn diagram depicting the prevalence and overlap of physical frailty, depressive symptoms and cognitive impairment in our cohort.

Table 1. Baseline clinical and demographic data. Asterisks $\left({ }^{*}\right)$ indicate significant difference between patients with and without physical frailty; hashes (\#) indicate significant difference between those with and without cognitive impairment

\begin{tabular}{|c|c|c|c|c|c|c|c|}
\hline \multirow{2}{*}{ Variable } & \multirow[b]{2}{*}{ all patients } & \multicolumn{2}{|c|}{ physical frailty } & \multicolumn{2}{|c|}{ depressive symptoms } & \multicolumn{2}{|c|}{ cognitive impairment } \\
\hline & & present & absent & present & absent & present & absent \\
\hline No. of patients & 178 & 111 & 67 & 49 & 129 & 14 & 164 \\
\hline Sex $(M: F)$ & 102:76 & $63: 48$ & $39: 28$ & $29: 20$ & $73: 56$ & $12: 2$ & $90: 74^{\#}$ \\
\hline Age (years) & $60.8 \pm 11.8$ & $62.1 \pm 11.4$ & $58.5 \pm 12.1^{*}$ & $62.1 \pm 11.5$ & $60.3 \pm 11.8$ & $72.5 \pm 8.8$ & $59.8 \pm 11.4^{\#}$ \\
\hline Duration of dialysis (months) & $42.8 \pm 44.1$ & $47.9 \pm 50.4$ & $34.3 \pm 29.3^{*}$ & $48.5 \pm 53.8$ & $40.6 \pm 39.8$ & $49.3 \pm 32.4$ & $42.2 \pm 45.0$ \\
\hline Body height $(\mathrm{cm})$ & $161.4 \pm 8.9$ & $160.4 \pm 8.7$ & $163.0 \pm 9.0$ & $160.3 \pm 9.1$ & $161.8 \pm 8.8$ & $162.1 \pm 6.5$ & $161.4 \pm 9.1$ \\
\hline Body weight $(\mathrm{kg})$ & $66.1 \pm 13.8$ & $66.0 \pm 13.2$ & $66.2 \pm 14.8$ & $65.0 \pm 12.5$ & $66.5 \pm 14.2$ & $67.6 \pm 10.3$ & $65.9 \pm 14.0$ \\
\hline \multicolumn{8}{|l|}{ Blood pressure $(\mathrm{mmHg})$} \\
\hline Systolic & $145.4 \pm 20.2$ & $143.6 \pm 19.7$ & $148.6 \pm 20.9$ & $142.5 \pm 22.3$ & $146.6 \pm 19.3$ & $148.9 \pm 17.8$ & $145.2 \pm 20.4$ \\
\hline Diastolic & $77.3 \pm 12.1$ & $75.6 \pm 11.6$ & $80.2 \pm 12.4^{*}$ & $76.1 \pm 13.2$ & $77.8 \pm 11.7$ & $71.2 \pm 9.8$ & $77.8 \pm 12.2$ \\
\hline \multicolumn{8}{|l|}{ Renal diagnosis, no. of cases (\%) } \\
\hline Glomerulonephritis & $50(28.1 \%)$ & $28(25.2 \%)$ & $22(32.8 \%)$ & $12(24.5 \%)$ & $38(29.5 \%)$ & $1(7.1 \%)$ & $49(29.9 \%)$ \\
\hline Diabetic nephropathy & $70(39.3 \%)$ & $47(42.3 \%)$ & $23(34.3 \%)$ & $24(49.0 \%)$ & $46(35.7 \%)$ & $8(57.1 \%)$ & $62(37.8 \%)$ \\
\hline Hypertensive nephrosclerosis & $18(10.1 \%)$ & $12(10.8 \%)$ & $6(9.0 \%)$ & $5(10.2 \%)$ & $13(10.1 \%)$ & $2(14.3 \%)$ & $16(9.8 \%)$ \\
\hline Polycystic kidney & $8(4.5 \%)$ & $5(4.5 \%)$ & $3(4.5 \%)$ & $2(4.1 \%)$ & $6(4.7 \%)$ & 0 & $8(4.9 \%)$ \\
\hline Urological diseases & $10(5.6 \%)$ & $7(6.3 \%)$ & $3(4.5 \%)$ & $2(4.1 \%)$ & $8(6.2 \%)$ & $1(7.1 \%)$ & $9(5.5 \%)$ \\
\hline Others / unknown & $22(12.4 \%)$ & $12(10.8 \%)$ & $10(14.9 \%)$ & $4(8.2 \%)$ & $18(13.9 \%)$ & $2(14.2 \%)$ & $11(6.7 \%)$ \\
\hline \multicolumn{8}{|l|}{ Comorbid disease, no. of cases (\%) } \\
\hline Diabetes & $82(46.1 \%)$ & $53(47.7 \%)$ & $29(43.3 \%)$ & 27 (55.1\%) & $55(42.6 \%)$ & $8(57.1 \%)$ & $74(45.1 \%)$ \\
\hline Coronary heart disease & $32(18.0 \%)$ & $23(20.7 \%)$ & $9(13.4 \%)$ & $11(22.4 \%)$ & $21(16.3 \%)$ & $4(28.6 \%)$ & $28(17.1 \%)$ \\
\hline Cerebrovascular disease & $42(23.6 \%)$ & $31(27.9 \%)$ & $11(16.4 \%)$ & $16(32.7 \%)$ & $26(20.2 \%)$ & $5(35.7 \%)$ & $37(22.6 \%)$ \\
\hline Charlson's comorbidity index & $5.6 \pm 2.0$ & $5.8 \pm 2.2$ & $5.1 \pm 1.8^{*}$ & $6.0 \pm 2.2$ & $5.4 \pm 2.0$ & $7.3 \pm 1.6$ & $5.4 \pm 2.0^{\#}$ \\
\hline
\end{tabular}




\section{Kidney Blood Pressure Research}

Table 2. Baseline biochemical and nutritional data. D/P4, dialysate-to-plasma creatinine ratio at 4 hour; MTAC, mass transfer area coefficient of creatinine; LDL, low density lipoprotein; HDL, high density lipoprotein; NPNA, normalized protein nitrogen appearance; FEBM, fat-free edema-free body mass; SGA, subjective global assessment; MIS, malnutrition inflammation score. Asterisks $\left({ }^{*}\right)$ indicate significant difference between patients with and without physical frailty; hashes (\#) indicate significant difference between those with and without cognitive impairment

\begin{tabular}{|c|c|c|c|c|c|c|c|}
\hline \multirow{2}{*}{ Variable } & \multirow[b]{2}{*}{ all patients } & \multicolumn{2}{|c|}{ physical frailty } & \multicolumn{2}{|c|}{ depressive symptoms } & \multicolumn{2}{|c|}{ cognitive impairment } \\
\hline & & present & absent & present & absent & present & absent \\
\hline $\begin{array}{l}\text { No. of patients } \\
\text { body built }\end{array}$ & 178 & 111 & 67 & 49 & 129 & 14 & 164 \\
\hline waist circumference $(\mathrm{cm})$ & $95.9 \pm 11.6$ & $96.9 \pm 11.6$ & $94.2 \pm 11.4$ & $97.5 \pm 11.3$ & $95.3 \pm 11.7$ & $96.6 \pm 8.6$ & $95.8 \pm 11.8$ \\
\hline hip circumference $(\mathrm{cm})$ & $99.7 \pm 9.8$ & $101.0 \pm 10.5$ & $97.7 \pm 8.4^{*}$ & $101.0 \pm 9.4$ & $99.3 \pm 10.0$ & $98.8 \pm 15.7$ & $99.7 \pm 9.4$ \\
\hline waist-hip ratio & $0.96 \pm 0.08$ & $0.96 \pm 0.10$ & $0.96 \pm 0.06$ & $0.96 \pm 0.09$ & $0.96 \pm 0.08$ & $1.01 \pm 0.21$ & $0.96 \pm 0.07$ \\
\hline \multicolumn{8}{|l|}{ peritoneal transport } \\
\hline $\mathrm{D} / \mathrm{P} 4$ & $0.63 \pm 0.13$ & $0.64 \pm 0.13$ & $0.62 \pm 0.14$ & $0.65 \pm 0.14$ & $0.63 \pm 0.13$ & $0.66 \pm 0.10$ & $0.63 \pm 0.14$ \\
\hline MTAC $(\mathrm{ml} / \mathrm{min} / 1.73 \mathrm{~m} 2)$ & $9.3 \pm 4.5$ & $9.5 \pm 4.7$ & $8.9 \pm 4.1$ & $10.0 \pm 5.4$ & $9.0 \pm 4.1$ & $9.5 \pm 3.3$ & $9.3 \pm 4.6$ \\
\hline hemoglobin $(\mathrm{g} / \mathrm{dL})$ & $9.3 \pm 1.2$ & $9.2 \pm 1.3$ & $9.4 \pm 1.2$ & $9.3 \pm 1.1$ & $9.3 \pm 1.3$ & $9.4 \pm 1.2$ & $9.3 \pm 1.3$ \\
\hline serum albumin (g/L) & $34.7 \pm 5.2$ & $34.0 \pm 4.9$ & $35.8 \pm 5.6$ & $33.1 \pm 5.3$ & $35.3 \pm 5.0^{\$}$ & $32.7 \pm 4.7$ & $34.9 \pm 5.2$ \\
\hline \multicolumn{8}{|l|}{ lipid profile (mmol/l) } \\
\hline total cholesterol & $5.0 \pm 2.1$ & $5.1 \pm 2.5$ & $5.0 \pm 2.5$ & $5.1 \pm 3.4$ & $5.0 \pm 1.4$ & $5.1 \pm 1.6$ & $5.0 \pm 2.1$ \\
\hline triglyceride & $1.6 \pm 1.0$ & $1.5 \pm 0.8$ & $1.7 \pm 1.2$ & $1.4 \pm 0.9$ & $1.6 \pm 1.0$ & $1.5 \pm 0.8$ & $1.6 \pm 1.0$ \\
\hline LDL cholesterol & $2.9 \pm 1.1$ & $2.8 \pm 1.1$ & $2.9 \pm 1.1$ & $2.5 \pm 0.9$ & $3.0 \pm 1.2^{\$}$ & $3.1 \pm 1.6$ & $2.8 \pm 1.1$ \\
\hline HDL cholesterol & $1.4 \pm 1.4$ & $1.4 \pm 0.7$ & $1.6 \pm 2.1$ & $1.4 \pm 0.9$ & $1.5 \pm 1.6$ & $1.8 \pm 1.8$ & $1.4 \pm 1.4$ \\
\hline total Kt/V & $1.83 \pm 0.49$ & $1.80 \pm 0.47$ & $1.89 \pm 0.51$ & $1.87 \pm 0.53$ & $1.82 \pm 0.47$ & $1.71 \pm 0.44$ & $1.84 \pm 0.49$ \\
\hline residual GFR $(\mathrm{ml} / \mathrm{min} / 1.73 \mathrm{~m} 2)$ & $1.89 \pm 2.37$ & $1.54 \pm 2.04$ & $2.46 \pm 2.76^{*}$ & $1.65 \pm 2.30$ & $1.97 \pm 2.41$ & $1.50 \pm 2.16$ & $1.92 \pm 2.40$ \\
\hline NPNA (g/kg/day) & $1.13 \pm 0.22$ & $1.10 \pm 0.21$ & $1.19 \pm 0.23^{*}$ & $1.11 \pm 0.22$ & $1.14 \pm 0.22$ & $1.01 \pm 0.24$ & $1.15 \pm 0.22$ \\
\hline FEBM $(\%)$ & $51.1 \pm 14.4$ & $52.0 \pm 13.5$ & $49.7 \pm 15.8$ & $51.0 \pm 13.6$ & $51.2 \pm 14.8$ & $47.8 \pm 9.3$ & $51.4 \pm 14.8$ \\
\hline overall SGA score & $5.44 \pm 0.70$ & $5.25 \pm 0.69$ & $5.75 \pm 0.61^{*}$ & $5.04 \pm 0.76$ & $5.59 \pm 0.62^{\$}$ & $5.29 \pm 0.61$ & $5.45 \pm 0.71$ \\
\hline MIS score & $7.01 \pm 3.58$ & $8.14 \pm 3.73$ & $5.12 \pm 2.30^{*}$ & $9.45 \pm 3.93$ & $6.08 \pm 2.95^{\$}$ & $9.00 \pm 2.86$ & $6.84 \pm 3.59 \#$ \\
\hline \multicolumn{8}{|l|}{ bioimpedance spectroscopy } \\
\hline overhydration (L) & $3.0 \pm 2.8$ & $3.2 \pm 2.5$ & $2.7 \pm 3.3$ & $3.7 \pm 2.8$ & $2.7 \pm 2.8^{\$}$ & $2.6 \pm 1.9$ & $3.0 \pm 2.9$ \\
\hline extracellular water (L) & $17.1 \pm 4.3$ & $17.1 \pm 4.1$ & $17.1 \pm 4.7$ & $17.4 \pm 4.5$ & $17.0 \pm 4.2$ & $16.0 \pm 1.8$ & $17.2 \pm 4.4$ \\
\hline lean tissue mass (kg) & $38.8 \pm 11.0$ & $37.2 \pm 10.3$ & $41.4 \pm 11.8^{*}$ & $37.7 \pm 11.5$ & $39.2 \pm 10.9$ & $32.1 \pm 5.9$ & $39.3 \pm 11.2^{\#}$ \\
\hline adipose tissue mass (kg) & $21.0 \pm 11.0$ & $21.7 \pm 10.4$ & $19.9 \pm 12.0$ & $19.5 \pm 9.2$ & $21.6 \pm 11.6$ & $28.6 \pm 9.2$ & $20.4 \pm 11.0^{\#}$ \\
\hline
\end{tabular}

\section{Additive effects of frailty and depressive symptoms}

Amongst the 111 patients with physical frailty, 48 (43.2\%) also had depressive symptoms, while most patients with depressive symptoms were also physically frail. We therefore divided the patients into 3 groups for further analysis: no frailty, frail but no depressive symptoms, and frail with depressive symptoms. Their nutritional status are summarized and compared in Table 3. In essence, there was an additive adverse effect of depressive symptoms and physical frailty on nutritional status in terms of serum albumin level, SGA score (and all its sub-scores), and MIS (and all its sub-scores except comorbidity and body mass index) (Fig. 2). The effect on NPNA did not reach statistical significance.

\section{Clinical outcome}

The patients were followed for $21.7 \pm 8.0$ months. During this period, 53 patients died. The causes of death were cardiovascular disease (20 cases), cerebrovascular diseases ( 3 cases), peritonitis ( 3 cases), non-peritonitis infections (19 cases), malignancy ( 1 case), liver cirrhosis ( 1 case), and termination of dialysis ( 1 case). During the same period, 9 patients were converted to long term hemodialysis, and 5 patients had kidney transplantation. Both patient survival and technique survival showed stepwise decrease for patients with no frailty, frail but no depressive symptoms, and frail with depressive symptoms. At 24 months, patient survival was $86.6 \%, 71.4 \%$, and $62.5 \%$ for the 3 groups respective (log rank test, $\mathrm{p}$ $=0.001)$, while the corresponding technique survival was $86.3 \%, 64.2 \%$, and $57.5 \%$ (p = 0.001) (Fig. 3).

During the same period, there were 751 hospital admissions for a total of 6418 days. Hospitalization also increased in a stepwise manner for patients with no frailty, frail but no depressive symptoms, and frail with depressive symptoms. The median number of hospital admission was 0.90 (IQR 0.44 - 2.06), 2.12 (IQR 0.91 - 3.74), and 3.31 (IQR 1.54 and 6.83) per year of follow up for the 3 groups respectively ( $<<0.0001$ ) (Fig. 4). Similarly, the median 


\section{Kidney Blood Pressure Research}

Table 3. Comparison of nutritional status between frail patients with and without depressive symptoms. LDL, low density lipoprotein; HDL, high density lipoprotein; NPNA, normalized protein nitrogen appearance; FEBM, fat-free edema-free body mass; SGA, subjective global assessment; MIS, malnutrition inflammation score; TIBC, total iron binding capacity

\begin{tabular}{|c|c|c|c|c|}
\hline patient group & not frail & no depressive symptoms & depressive symptoms & P value \\
\hline No. of patients & 67 & 63 & 48 & \\
\hline Charlson's score & $5.1 \pm 1.8$ & $5.7 \pm 2.2$ & $6.0 \pm 2.1$ & $\mathrm{p}=0.051$ \\
\hline \multicolumn{5}{|l|}{ body built } \\
\hline waist circumference $(\mathrm{cm})$ & $94.2 \pm 11.4$ & $96.4 \pm 12.0$ & $97.7 \pm 11.2$ & $\mathrm{p}=0.3$ \\
\hline hip circumference $(\mathrm{cm})$ & $97.7 \pm 8.4$ & $100.7 \pm 11.3$ & $101.4 \pm 9.2$ & $\mathrm{p}=0.10$ \\
\hline waist-hip ratio & $0.96 \pm 0.06$ & $0.96 \pm 0.10$ & $0.96 \pm 0.09$ & $\mathrm{p}=0.9$ \\
\hline hemoglobin $(\mathrm{g} / \mathrm{dL})$ & $9.4 \pm 1.2$ & $9.1 \pm 1.4$ & $9.3 \pm 1.1$ & $\mathrm{p}=0.5$ \\
\hline serum albumin $(\mathrm{g} / \mathrm{L})$ & $35.8 \pm 5.6$ & $34.9 \pm 4.4$ & $32.9 \pm 5.3$ & $p=0.025$ \\
\hline \multicolumn{5}{|l|}{ lipid profile $(\mathrm{mmol} / \mathrm{l})$} \\
\hline total cholesterol & $5.0 \pm 1.3$ & $5.1 \pm 1.4$ & $5.1 \pm 3.4$ & $\mathrm{p}=0.9$ \\
\hline triglyceride & $1.7 \pm 1.2$ & $1.5 \pm 0.7$ & $1.5 \pm 0.9$ & $\mathrm{p}=0.4$ \\
\hline LDL cholesterol & $2.9 \pm 1.1$ & $3.0 \pm 1.2$ & $2.6 \pm 0.9$ & $\mathrm{p}=0.06$ \\
\hline HD cholesterol & $1.6 \pm 2.1$ & $1.3 \pm 0.5$ & $1.4 \pm 0.9$ & $\mathrm{p}=0.5$ \\
\hline total Kt/V & $1.89 \pm 0.51$ & $1.78 \pm 0.49$ & $1.83 \pm 0.45$ & $\mathrm{p}=0.5$ \\
\hline residual GFR (ml/min/1.73m2) & $2.46 \pm 2.76$ & $1.56 \pm 1.99$ & $1.51 \pm 2.13$ & $\mathrm{p}=0.07$ \\
\hline NPNA (g/kg/day) & $1.19 \pm 0.23$ & $1.11 \pm 0.22$ & $1.10 \pm 0.21$ & $\mathrm{p}=0.09$ \\
\hline FEBM $(\%)$ & $49.7 \pm 15.8$ & $52.5 \pm 13.5$ & $51.3 \pm 13.6$ & $\mathrm{p}=0.6$ \\
\hline \multicolumn{5}{|l|}{ overall SGA score } \\
\hline weight loss & $5.94 \pm 0.69$ & $5.63 \pm 0.77$ & $5.40 \pm 0.96$ & $\mathrm{p}=0.002$ \\
\hline anorexia & $5.87 \pm 0.58$ & $5.33 \pm 0.76$ & $5.08 \pm 0.94$ & $\mathrm{p}<0.0001$ \\
\hline subcutaneous fat & $5.55 \pm 0.88$ & $5.43 \pm 0.76$ & $5.08 \pm 0.85$ & $\mathrm{p}=0.011$ \\
\hline muscle mass & $5.46 \pm 0.70$ & $5.22 \pm 0.79$ & $4.96 \pm 0.68$ & $\mathrm{p}=0.002$ \\
\hline overall score & $5.75 \pm 0.61$ & $5.41 \pm 0.59$ & $5.04 \pm 0.77$ & $\mathrm{p}<0.0001$ \\
\hline \multicolumn{5}{|l|}{ MIS score } \\
\hline change in dry weight & $0.33 \pm 0.81$ & $0.65 \pm 0.97$ & $0.83 \pm 1.06$ & $\mathrm{p}=0.017$ \\
\hline dietary intake & $0.16 \pm 0.37$ & $0.33 \pm 0.48$ & $0.58 \pm 0.58$ & $\mathrm{p}<0.0001$ \\
\hline gastrointestinal symptom & $0.15 \pm 0.36$ & $0.49 \pm 0.64$ & $0.75 \pm 0.79$ & $\mathrm{p}<0.0001$ \\
\hline functional capacity & $0.12 \pm 0.33$ & $0.56 \pm 0.71$ & $0.96 \pm 0.82$ & $\mathrm{p}<0.0001$ \\
\hline comorbidity & $0.96 \pm 0.68$ & $1.17 \pm 0.71$ & $1.17 \pm 0.69$ & $\mathrm{p}=0.14$ \\
\hline loss of subcutaneous fat & $0.48 \pm 0.68$ & $0.51 \pm 0.64$ & $0.75 \pm 0.79$ & $\mathrm{p}=0.09$ \\
\hline muscle wasting & $0.55 \pm 0.07$ & $0.71 \pm 0.09$ & $0.73 \pm 0.11$ & $\mathrm{p}=0.001$ \\
\hline body mass index & $0.07 \pm 0.27$ & $0.10 \pm 0.35$ & $0.06 \pm 0.32$ & $\mathrm{p}=0.9$ \\
\hline serum albumin & $1.33 \pm 0.77$ & $1.51 \pm 0.95$ & $1.96 \pm 1.05$ & $p=0.001$ \\
\hline serum TIBC & $1.13 \pm 0.76$ & $1.22 \pm 0.85$ & $1.56 \pm 0.94$ & $\mathrm{p}=0.02$ \\
\hline total score & $5.12 \pm 2.30$ & $7.13 \pm 3.22$ & $9.48 \pm 3.97$ & $\mathrm{p}<0.0001$ \\
\hline \multicolumn{5}{|l|}{ bioimpedance spectroscopy } \\
\hline overhydration (L) & $2.7 \pm 3.3$ & $2.8 \pm 2.1$ & $3.9 \pm 2.8$ & $\mathrm{p}=0.08$ \\
\hline extracellular water (L) & $17.1 \pm 4.7$ & $16.8 \pm 3.8$ & $17.6 \pm 4.4$ & $\mathrm{p}=0.7$ \\
\hline lean tissue mass (kg) & $41.4 \pm 11.8$ & $36.8 \pm 9.3$ & $37.8 \pm 11.5$ & $\mathrm{p}=0.06$ \\
\hline adipose tissue mass $(\mathrm{kg})$ & $19.9 \pm 12.0$ & $23.2 \pm 11.1$ & $19.7 \pm 9.2$ & $\mathrm{p}=0.2$ \\
\hline
\end{tabular}

Fig. 2. Comparison of nutritional status amongst the patients with no frailty, frail but no depressive symptoms, and frail with depressive symptoms: (A) serum albumin level; (B) normalized protein nitrogen appearance (NPNA); (C) overall subjective global assessment (SGA) score; and (D) malnutrition inflammation score (MIS). Overall comparison was performed by one way analysis of variance (ANOVA). Post hoc subgroup analysis was performed by unpaired Student's t test, with $\mathrm{p}$ values corrected by the Bonferroni method for multiple comparisons.
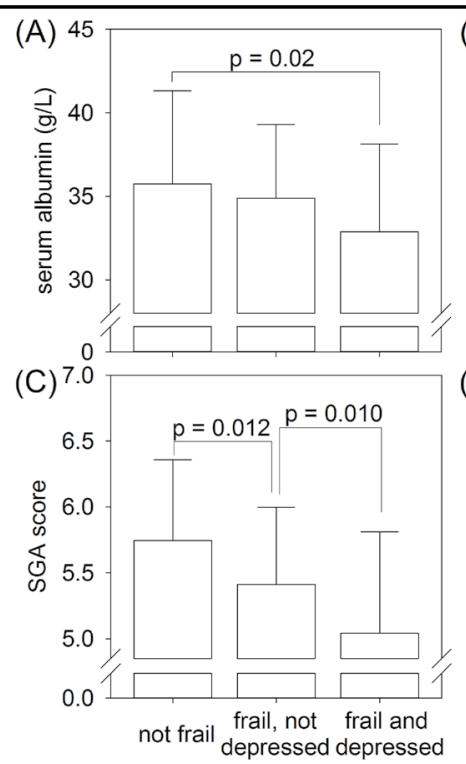

(D) 16
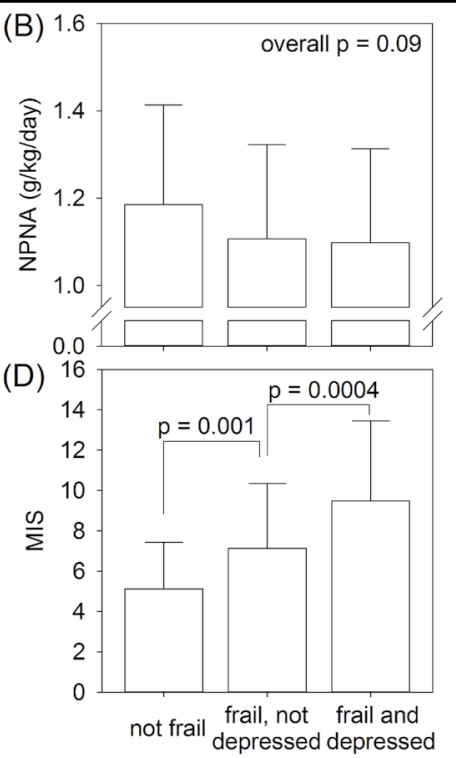


\section{Kidney Blood Pressure Research}

Fig. 3. Kaplan Meier plot for: (A) patient survival; and (B) technique survival. Patients were divided into three groups: no frailty, frail but no depressive symptoms, and frail with depressive symptoms. Data were compared by the log rank test.

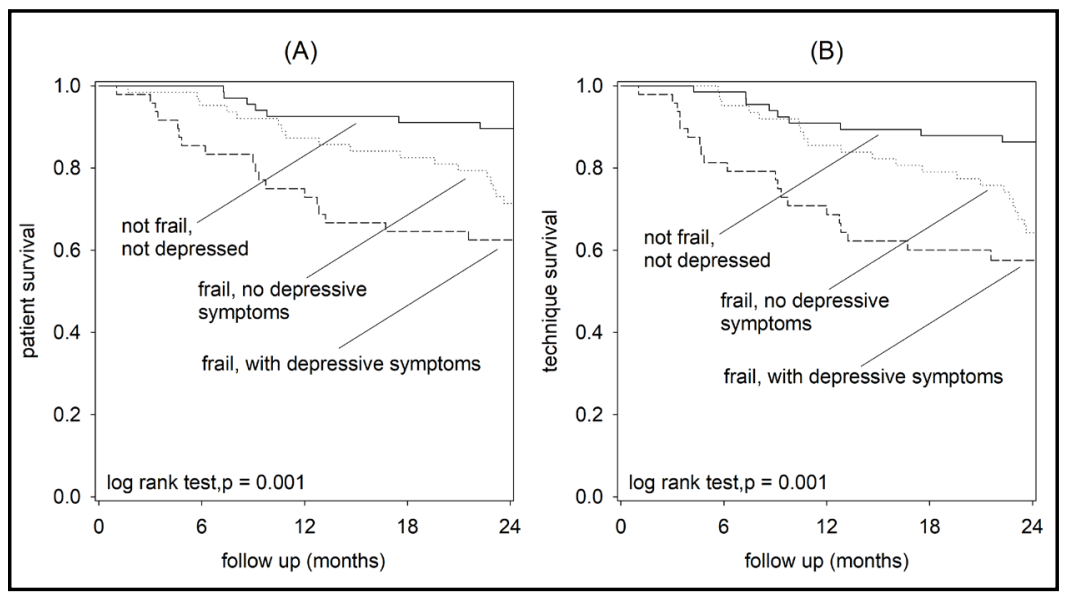

Fig. 4. Comparison of (A) number of hospital admission; and (B) duration of hospitalization amongst the patients with no frailty, frail but no depressive symptoms, and frail with depressive symptoms. Whisker-box plot, with the boxes indicate median, 25 and 75 percentile; whisker caps indicate 5 and 95 percentile; circles indicate outliers. Overall $\mathrm{p}<0.0001$ for both comparisons by Kruskal Wallis test; $\mathrm{p}$ values depicted on the Fig. represent post hoc analysis by MannWhitney U test, with p values corrected by the Bonferroni method for multiple comparison.

number of hospital stay was 8.04 (IQR 0.91 - 19.42), 14.05 (IQR 3.57 - 37.27), and 26.62 (IQR 10.65 - 61.18) days per year of follow up, respectively ( $<0.0001$ ) (Fig. 4).

\section{Discussion}

In the present study, we find that physical frailty, depressive symptoms, and cognitive impairment are all associated with a worse nutritional status in Chinese PD patients. More importantly, our result indicates that depressive symptoms and physical frailty have additive adverse effect on the nutritional status and clinical outcome in this group of patients.

Our findings are consistent with previous reports but provide new insight to the interaction between physical frailty and depression in dialysis patients. In the non-dialysis elderly general population, depression and frailty are additive risk factors for mortality and other adverse health outcomes [18-20]. In previous studies on dialysis patients, physical frailty $[6,7]$ and depression $[12,13]$ have each been shown to be associated with adverse clinical outcomes. Our results show that their effects in PD patients are also additive.

The prevalence of physical frailty in our PD population is similar to the published literature. For example, previous studies found that physical frailty is present in around $35 \%$ to $50 \%$ of hemodialysis patients $[8,32]$ and over $60 \%$ of PD patients [23], which are similar to the prevalence of $62 \%$ in our present study. On the other hand, the prevalence of depression 


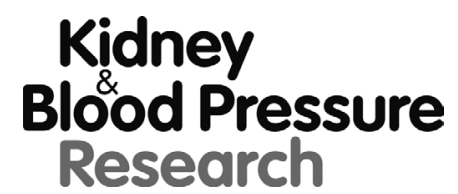

\begin{tabular}{l|l}
\hline Kidney Blood Press Res 2018;43:914-923 \\
\hline DOI: 10.1159/000490470 & $\begin{array}{l}\text { c } 2018 \text { The Author(s). Published by S. Karger AG, Basel } \\
\text { www.karger.com/kbr }\end{array}$ \\
\hline Published onlIne: I2 June, 2018 &
\end{tabular}

Szeto et al.: Depression and Frailty in PD

and cognitive impairment are considerably lower in our present study than previous ones. Notably, depressive symptoms are present in $27.5 \%$ patients in this study, but in over $50 \%$ of Chinese PD patients in previous reports [10,33]. Similarly, cognitive impairment is present in over $20 \%$ of patients receiving hemodialysis [9] as well as PD [10], but is less than $8 \%$ in the present study. The cause of this discrepancy in prevalence is unknown, but may reflect the difference in background comorbidity load and eligibility criteria of dialysis across different countries. The implication is one should be cautious to extrapolate our result to other patient population. Moreover, since the prevalence of cognitive impairment is low in our cohort, we study does not have sufficient power to determine any interaction between cognitive impairment and physical frailty or depressive symptoms.

Our result also highlights the delicate difference in body composition between PD patients with and without physical frailty, and the problem of using body weight as a marker of malnutrition. Specifically, although the crude body weight was similar between patients with and without physical frailty, the former group had significantly lower lean tissue mass but higher adipose tissue mass (Table 2), indicating concomitant muscle atrophy and accumulation of body fat due to the lack of physical activity. Previous study showed that frailty exacerbates protein catabolism and loss of muscle mass [34], which is related to the cardiovascular and overall mortality in the elderly population [35]. To the best of our knowledge, alterations of adipose tissue mass in frail elderly patients have not been reported previously. Our results show that the loss of muscle mass in frail PD patients is often masked by the increase in body fat.

There are several inadequacies of our study. Although our sample size was considerable, we had only one baseline assessment for the presence of physical frailty, depressive symptoms, cognitive impairment, or nutritional status. As a result, we cannot determine whether there is any temporal relationship between physical frailty and depressive symptoms, or whether they would cause progressive worsening of nutritional status or body composition. In this regard, a recent systematic review found a consistent relationship between depression and new onset physical frailty [21], and frailty is associated with the progressive loss of muscle mass in patients without renal disease [34]. Further studies are needed to determine the effect of physical frailty and depressive symptoms on the longitudinal change in nutritional status and body composition in PD patients.

We did not perform sophisticated multivariate analysis to determine whether physical frailty and depressive symptoms independently contribute to malnutrition. In addition, we could not ascertain whether physical frailty and depressive symptoms are directly responsible for the adverse clinical outcome (i.e. excessive mortality and higher hospitalization rate), or that the adverse clinical outcomes were the result of malnutrition secondary to physical frailty and depressive symptoms. Further studies with much bigger sample size are needed to have the sufficient statistical power for a robust multivariate analysis that could discern the role of malnutrition in the adverse clinical outcomes associated with physical frailty and depressive symptoms, or whether therapeutic interventions (for example, physical training, anti-depressant drugs, or nutritional supplement) could improve the outcome of these patients. Notably, a recent study showed that physical training during dialysis, in the terms of both resistance and endurance training, is beneficial in reducing depressive symptoms of dialysis patients with end stage renal disease [36].

\section{Conclusion}

We show that physical frailty and depressive symptoms are common in Chinese PD patients and they have additive adverse effect on the nutritional status and clinical outcome. Our results suggest that there are two groups of frail PD patients - those with and without depressive symptoms, and depressive symptoms are usually absent without frailty. 


\section{Kidney Blood Pressure Research}

Szeto et al.: Depression and Frailty in PD

\section{Acknowledgements}

This study was supported by the Chinese University of Hong Kong (CUHK) research accounts 6901031 and 7101215. The funders had no role in study design, data collection and analysis, decision to publish, or preparation of the manuscript. Dr. CC Szeto receives research grant and consultancy from Baxter Healthcare.

\section{Disclosure Statement}

The authors declare no other conflicts of interest.

The results presented in this paper have not been published previously in whole or part, except in abstract format.

\section{References}

1 Jain AK, Blake P, Cordy P, Garg AX: Global trends in rates of peritoneal dialysis. J Am Soc Nephrol 2012;23:533-544.

2 Perl J, Wald R, Bargman JM, Na Y, Jassal SV, Jain AK, Moist L, Nessim SJ: Changes in patient and technique survival over time among incident peritoneal dialysis patients in Canada. Clin J Am Soc Nephrol 2012;7:1145-1154.

- 3 Davies SJ: Peritoneal dialysis-current status and future challenges. Nat Rev Nephrol 2013;9:399-408.

4 Seidel UK, Gronewold J, Volsek M, Todica O, Kribben A, Bruck H, Hermann DM: Physical, cognitive and emotional factors contributing to quality of life, functional health and participation in community dwelling in chronic kidney disease. PLoS One 2014;9:e91176.

5 van Loon IN, Wouters TR, Boereboom FT, Bots ML, Verhaar MC, Hamaker ME: The Relevance of Geriatric Impairments in Patients Starting Dialysis: A Systematic Review. Clin J Am Soc Nephrol 2016;11:1245-1159.

6 Xue QL: The frailty syndrome: definition and natural history. Clin Geriatr Med 2011;27:1-15.

7 Fried LP, Tangen CM, Walston J, Newman AB, Hirsch C, Gottdiener J, Seeman T, Tracy R, Kop WJ, Burke G, McBurnie MA: Frailty in older adults: evidence for a phenotype. J Gerontol A Biol Sci Med Sci 2001;56:M146-M156.

8 McAdams-DeMarco MA, Law A, Salter ML, Boyarsky B, Gimenez L, Jaar BG, Walston JD, Segev DL: Frailty as a novel predictor of mortality and hospitalization in individuals of all ages undergoing hemodialysis. J Am Geriatr Soc 2013;61:896-901.

-9 Kallenberg MH, Kleinveld HA, Dekker FW, van Munster BC, Rabelink TJ, van Buren M, Mooijaart SP: Functional and Cognitive Impairment, Frailty, and Adverse Health Outcomes in Older Patients Reaching ESRD-A Systematic Review. Clin J Am Soc Nephrol. 2016;11:1624-1639.

10 Dong J, Pi HC, Xiong ZY, Liao JL, Hao L, Liu GL, Ren YP, Wang Q Duan LP, Zheng ZX: Depression and Cognitive Impairment in Peritoneal Dialysis: A Multicenter Cross-sectional Study. Am J Kidney Dis 2016;67:111-118.

-11 Drew DA, Weiner DE, Tighiouart H, Scott T, Lou K, Kantor A, Fan L, Strom JA, Singh AK, Sarnak MJ: Cognitive function and all-cause mortality in maintenance hemodialysis patients. Am J Kidney Dis 2015;65:303-311.

12 Turkmen K, Guney I, Yazici R, Arslan S, Altintepe L, Yeksan M: Health-related quality of life, depression and mortality in peritoneal dialysis patients in Turkey: seven-year experience of a center. Ren Fail 2014;36:859-864.

13 Szeto CC: Peritoneal dialysis-related infection in the older population. Perit Dial Int 2015;35:659-662.

14 Halil M, Cemal Kizilarslanoglu M, Emin Kuyumcu M, Yesil Y, Cruz Jentoft AJ: Cognitive aspects of frailty: mechanisms behind the link between frailty and cognitive impairment. J Nutr Health Aging 2015;19:276283.

15 McAdams-DeMarco MA, Tan J, Salter ML, Gross A, Meoni LA, Jaar BG, Kao WH, Parekh RS, Segev DL, Sozio SM: Frailty and Cognitive Function in Incident Hemodialysis Patients. Clin J Am Soc Nephrol 2015;10:21812189. 


\section{Kidney \\ Bloód Pressure Research}

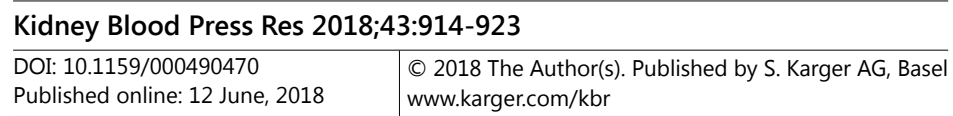

Szeto et al.: Depression and Frailty in PD

16 Boyle PA, Buchman AS, Wilson RS, Leurgans SE, Bennett DA: Physical frailty is associated with incident mild cognitive impairment in community-based older persons. J Am Geriatr Soc 2010;58:248-255.

17 Searle SD, Rockwood K: Frailty and the risk of cognitive impairment. Alzheimers Res Ther 2015;7:54.

18 Lohman MC, Mezuk B, Dumenci L: Depression and frailty: concurrent risks for adverse health outcomes. Aging Ment Health 2015;19:1-10.

19 Almeida OP, Hankey GJ, Yeap BB, Golledge J, Norman PE, Flicker L: Depression, frailty, and all-cause mortality: a cohort study of men older than 75 years. J Am Med Dir Assoc 2015;16:296-300.

-20 Brown PJ, Roose SP, Fieo R, Liu X, Rantanen T, Sneed JR, Rutherford BR, Devanand DP, Avlund K: Frailty and depression in older adults: a high-risk clinical population. Am J Geriatr Psychiatry 2014;22:1083-1095.

-21 Vaughan L, Corbin AL, Goveas JS: Depression and frailty in later life: a systematic review. Clin Interv Aging 2015;10:1947-1958.

22 Beddhu S, Zeidel ML, Saul M, Seddon P, Samore MH, Stoddard GJ, Bruns FJ: The effects of comorbid conditions on the outcomes of patients undergoing peritoneal dialysis. Am J Med 2002;112:696-701.

23 Ng JK, Kwan BC, Chow KM, Cheng PM, Law MC, Pang WF, Leung CB, Li PK, Szeto CC: Frailty in Chinese Peritoneal Dialysis Patients: Prevalence and Prognostic Significance. Kidney Blood Press Res 2016;41:736745.

24 Balogun RA, Balogun SA, Kepple AL, Ma JZ, Turgut F, Kovesdy CP, Abdel-Rahman EM: The 15-item geriatric depression scale as a predictor of mortality in older adults undergoing hemodialysis. J Am Geriatr Soc 2011;59:1563-1565.

25 Lam SC, Wong YY, Woo J: Reliability and validity of the abbreviated mental test (Hong Kong version) in residential care homes. J Am Geriatr Soc 2010;58: 2255-2257.

26 Szeto CC, Wong TY, Chow KM, Leung CB, Li PK: Oral sodium bicarbonate for the treatment of metabolic acidosis in peritoneal dialysis patients-a randomized placebo-control trial. J Am Soc Nephrol 2003;14:2119-2126.

-27 Enia G, Sicus C, Alati G, Zoccali C: Subjective global assessment of nutrition in dialysis patients. Nephrol Dial Transplant 1993;8:1094-1098.

28 Kalantar-Zadeh K, Kopple JD, Block G, Humphreys MH: A malnutrition-inflammation score is correlated with morbidity and mortality in maintenance hemodialysis patients. Am J Kidney Dis 2001;38:1251-1263.

-29 Bergstrom J, Heimburger 0, Lindholm B: Calculation of the protein equivalent of total nitrogen appearance from urea appearance. Which formulas should be used? Perit Dial Int 1998;18:467-473.

-30 Kwan BC, Szeto CC, Chow KM, Law MC, Cheng MS, Leung CB, Pang WF, Kwong VW, Li PK: Bioimpedance spectroscopy for the detection of fluid overload in Chinese peritoneal dialysis patients. Perit Dial Int 2014;34:409-416.

-31 Choy AS, Li PK: Sustainability of the Peritoneal Dialysis-First Policy in Hong Kong. Blood Purif 2015;40:320-325.

32 Musso CG, Jauregui JR, Macías Núñez JF: Frailty phenotype and chronic kidney disease: a review of the literature. Int Urol Nephrol 2015;47:1801-1807.

-33 Chan KM, Cheung CY, Chan YH, Chan HW, Chak WL, Chau KF: Prevalence and impact of anxiety and depression in Chinese peritoneal dialysis patients: a single center study. Nephrology (Carlton) 2018;23:155-161.

-34 Chevalier S, Gougeon R, Nayar K, Morais JA. Frailty amplifies the effects of aging on protein metabolism: role of protein intake: Am J Clin Nutr 2003;78:422-429.

35 Spahillari A, Mukamal KJ, DeFilippi C, Kizer JR, Gottdiener JS, Djoussé L, Lyles MF, Bartz TM, Murthy VL, Shah RV: The association of lean and fat mass with all-cause mortality in older adults: The Cardiovascular Health Study. Nutr Metab Cardiovasc Dis 2016;26:1039-1047.

-36 Dziubek W, Kowalska J, Kusztal M, Rogowski Ł, Gołębiowski T, Nikifur M, Szczepańska-Gieracha J, Zembroń-Łacny A, Klinger M, Woźniewski M: The Level of Anxiety and Depression in Dialysis Patients Undertaking Regular Physical Exercise Training-a Preliminary Study. Kidney Blood Press Res 2016;41:8698. 